\title{
Entretien avec Loïc Touzé : « Amplifier le désastre par la tentative du sauvetage même »
}

\section{Loïc Touzé et Anne Pellois}

\section{(2) OpenEdition \\ Journals}

\section{Édition électronique}

URL : https://journals.openedition.org/agon/8725

DOI : $10.4000 /$ agon. 8725

ISSN : 1961-8581

Éditeur

Association Agôn

Référence électronique

Loïc Touzé et Anne Pellois, «Entretien avec Loïc Touzé : «Amplifier le désastre par la tentative du sauvetage même » », Agôn [En ligne], 9 | 2021, mis en ligne le 17 novembre 2021, consulté le 18 janvier 2022. URL : http://journals.openedition.org/agon/8725 ; DOI : https://doi.org/10.4000/agon.8725

Ce document a été généré automatiquement le 18 janvier 2022.

Association Agôn et les auteurs des articles 


\title{
Entretien avec Loïc Touzé : «Amplifier le désastre par la tentative du sauvetage même »
}

\author{
Loïc Touzé et Anne Pellois
}

\section{NOTE DE L'ÉDITEUR}

\section{Entretien réalisé le 02 juillet 2020}

Anne Pellois: Je te propose d'aborder la question du ratage en fonction de toutes tes casquettes: chorégraphe, interprète et pédagogue. Et je te propose aussi d'envisager les deux aspects du ratage: positif et négatif, car je ne doute pas que tu auras évidemment, connaissant ton travail, un rapport positif au ratage! Je te propose trois définitions sur lesquelles nous pourrions nous accorder ou faire bouger au cours de l'entretien :
1. Ne pas partir, ne pas avoir lieu, avorter. Rater c'est donc un faux départ, une absence d'événement. Il est prévu que quelque chose se passe, et il ne se passe rien. Le verbe est utilisé au départ pour désigner un coup de feu qui ne part pas.
2. Ne pas réussir. Ne pas obtenir le résultat souhaité : il se passe quelque chose, mais ce n'est pas la chose attendue.
3. Manquer l'occasion, la rencontre, laisser passer quelque chose qui aurait pu advenir d'une relation et qui ne se passe pas.

Le terme induit ainsi une forme de déficience, et une forme de maladresse, ou encore un manque d'à-propos. Celui ou celle qui rate passe à côté de sa performance, soit parce que la performance n'advient pas, au sens 1, soit qu'elle n'est pas à la hauteur du résultat espéré, au sens 2 , soit enfin qu'elle ne saisit pas la bonne opportunité, au sens 3 . Nous verrons si ces définitions ou ces pistes tiennent tout au long de l'entretien.

As-tu déjà eu la sensation, en tant que chorégraphe et danseur, de rater un spectacle? Qu'est-ce qu'on rate quand on rate un spectacle? Est-ce que l'endroit du ratage est le même quand on est un danseur ou un chorégraphe?

Loïc Touzé : La première pièce que j'ai réalisée, je l'ai co-signée avec la danseuse Bernadette Doneux. Nous nous étions rencontrés dans la compagnie de Carolyn 
Carlson. Je venais de démissionner de l'Opéra de Paris où j'avais passé douze années, huit ans d'école de danse, puis quatre années dans la compagnie dont la dernière au sein du GRCOP ${ }^{1}$. J'ai alors très peu de connaissances en danse contemporaine. J'ai rencontré quelques chorégraphes et participé à des créations mais je n'ai encore aucune notion de ce qu'est la composition en danse, même si je vais quand même approcher différents processus de création. Bernadette a déjà créé un solo. Nous nous engageons ensemble dans le processus de composition de La chambre aux tournesols, pièce inspirée de la correspondance entre Vincent Van Gogh et de son frère Théo. Dans la naïveté qui est la nôtre à ce moment-là, nous restons très littéraux : la scénographie est un champ de blé en raffia, le fond de scène une toile peinte en jaune. Sur le plateau une table, une chaise, un lit. Tout est traité de façon figurative. Christian Ferry, programmateur, nous propose de présenter ce spectacle au théâtre de la Bastille à Paris. Nous sommes donc tout de suite propulsés dans un espace de grande visibilité. C'est l'endroit où il faut être pour présenter son travail en France à la fin des années 80 . Six représentations sont prévues dans la grande salle du théâtre. Nous sommes inquiets que la pièce ne soit pas suffisamment solide, nous allons tout faire pour nous sentir à la hauteur de l'invitation qui nous est faite. La scénographie, les costumes, la bande-son, tout l'habillage est surinvesti. La pièce est très formelle, son écriture chorégraphique très tenue, cela va faire un succès. Le public et les professionnels y reconnaissent là une danse dont ils ont envie. J'ai tout de suite conscience d'un ratage, je passe à côté d'une expérience et d'une expérimentation. Mais je ne suis pas, à ce moment-là, capable de faire autre chose. Je fais une pièce qui répond aux attentes de cette période. Par chance, j'ai des amis qui vont venir me dire que cela n'est pas bon du tout, malgré tous les signes du succès. Pendant les trois ans qui vont suivre, je choisis de suspendre mes velléités chorégraphiques et je trouve du travail en tant que danseur pour des chorégraphes contemporains. Ce sera le début de ma formation, observer comment font les autres. J'ai beaucoup de tendresse pour cette première pièce, mais je la considère comme un ratage.

\section{A. P. : C'est un échec, parce que ça n'a pas été la possibilité d'une expérience?}

L. T.: Nous allons passer notre temps à nous assurer que la pièce tienne dans son efficacité rythmique. Soigner les transitions, travailler l'unisson, mettre des ritournelles enfantines, ou de la musique lyrique rassurante et populaire avec des chants très émotionnels. Nos costumes sont peints avec des couleurs primaires du bleu, du rouge, du jaune. Nous nous engouffrons dans tous les clichés que la lecture de ces lettres inspirait. Nous ne voulons surtout pas être hors-sujet. Ce qui fait que nous tombons dans le commentaire et l'illustration. Jamais nous ne prendrons le risque d'une aventure plus figurale. Nous n'en sommes alors pas capables.

\section{A. P. : À l'inverse, as-tu eu le sentiment d'une réussite totale mais qui a raté son public ?}

L. T.: Au cours de ces trente dernières années, plusieurs des pièces que j'ai créées n'ont pas rencontré le public dans le sens d'une adhésion et pour autant je les considère comme réussies. Les raisons de cela sont de différentes natures. Parfois les pièces ne sont pas abouties mais le processus qui les a vu naître a été très stimulant. Parfois les professionnels-programmateurs, intermédiaires entre la pièce et le public, considèrent que ce n'est pas ce qu'il faut présenter. 1992-1999 est la période des études chorégraphiques. Je vais déconstruire tout ce que j'ai appris, me défaire d'un langage académique, imaginer de nouvelles façons d'envisager l'espace, sortir des scènes conventionnelles, me déplacer sur d'autres terrains pour comprendre 
comment faire un geste différent, chercher des nouveaux rapports entre ce qui est composé et la manière dont cela est perçu par le public. Ces tentatives sont une fois sur deux un échec mais sont utiles au processus de reconfiguration que j'engage à ce moment-là. À cette époque, il n'y avait pas d'écoles de chorégraphie. On se servait des moyens de production pour chercher, faire advenir un langage, expérimenter. J'ai mis beaucoup de temps à comprendre que je n'étais pas obligé de répondre aux attentes supposées des professionnels et du public. Avec Morceau en 2000, je renverse la table.

A. P. : Tu parles là du ratage en tant que chorégraphe, mais qu'est-ce que c'est que rater quand on est interprète? J'imagine que ce n'est pas la même chose quand on est interprète en danse classique et dans d'autres types de danse?

L. T.: Le besoin impérieux d'émancipation d'une culture académique que j'avais rencontrée très jeune à l'Opéra de Paris est lié à ma recherche d'un espace de ratage pour pouvoir grandir.

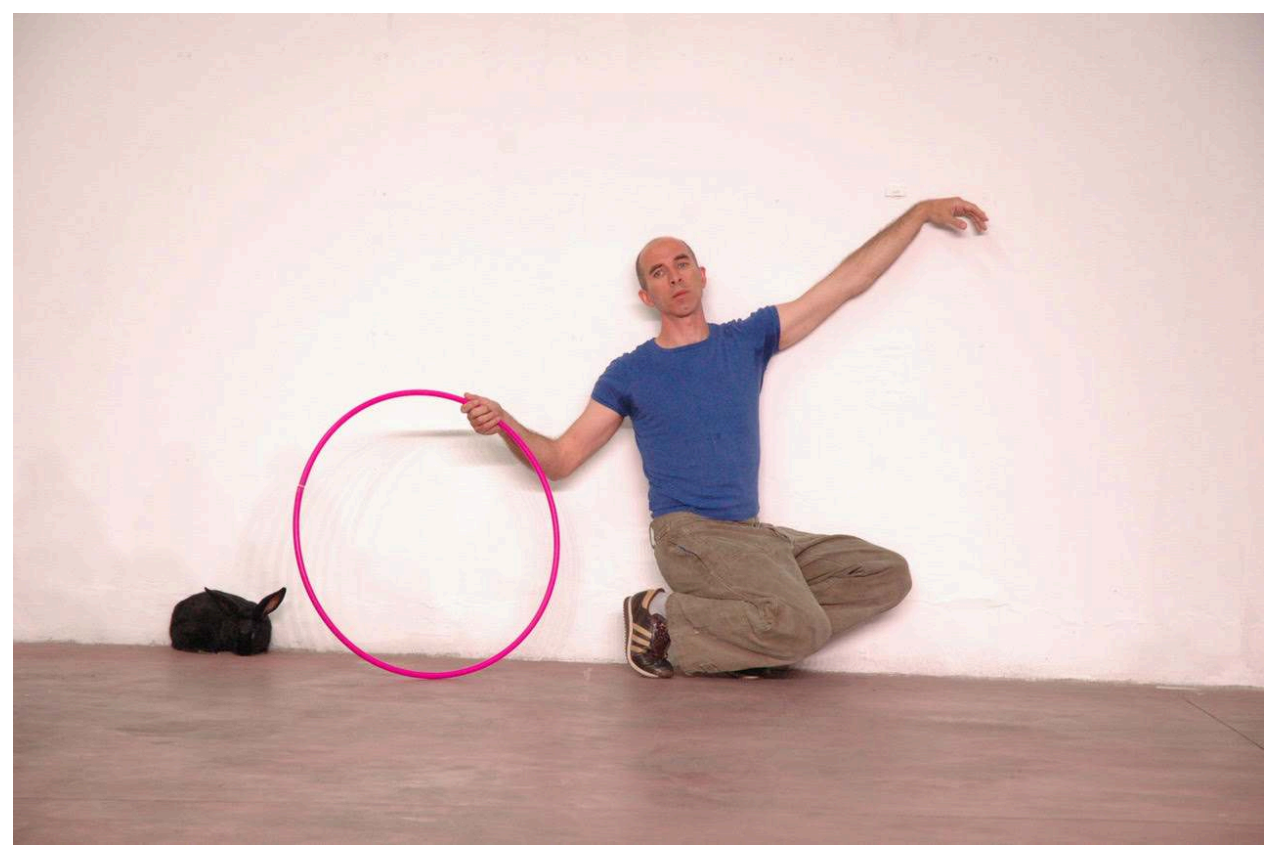

Loïc Touzé dans Morceau

(C) Cosimo Terlizzi

L'opéra est une école qui contient un vrai paradoxe : école de l'excellence où l'échec y tient la plus grande place. Un à deux pour cent des élèves vont devenir des professionnels et intégrer la compagnie, les autres seront rejetés, renvoyés. Une fois dans la compagnie, recommence un cycle de concours pour arriver à monter dans la hiérarchie. Entre 10 ans et 30 ans, tu passes ta vie à faire des concours pour pouvoir danser un rôle principal dans un ballet avec très peu de chances d'y parvenir étant donné le très haut niveau de concurrence.

J'ai très tôt compris que la réussite relevait plus du hasard que de la compétence, des circonstances plus que de l'habilité. Instinctivement j'ai saisi que la réussite était très improbable. J'ai alors décidé non pas de réussir mais de ne pas échouer. Je me suis débrouillé pour ne pas me faire renvoyer. Je n'étais pas assez doué pour passer facilement d'une classe à l'autre, j'avais besoin de plus de temps que les autres élèves. Dans un système où la règle est d'aller vite et de prouver très tôt ses capacités à 
réussir, j'ai résisté au rythme de l'institution, je me suis débrouillé pour, trois fois de suite, redoubler une classe. Cette école qui se fait en 5 ans, je l'ai faite en 8. J'ai pris mon temps, et en faisant cela j'ai construit un rapport critique au geste qui m'était inculqué, en lui opposant une certaine lenteur. C'est la lenteur de mon apprentissage qui m'a fait comprendre que je pouvais avoir mon rythme à moi, rater et par là même avoir une compréhension plus vaste du geste que j'engageais. Par ce temps pris, et ce ratage assumé, j'ai réussi à rentrer dans la compagnie. Je pense que ça a inscrit dans mon ADN de danseur que l'excellence était demandée mais que, pour pouvoir l'atteindre à ma manière, il fallait que j'y inclue la possibilité du ratage. Quand tu es danseur classique, il n'est pas question de rater une prouesse technique. Tu es jugé comme un patineur artistique. Il y a des figures à réaliser. Pour sortir du corps de ballet par le haut, la seule réussite, c'est l'excellence de ta technicité, parfois associée à un certain charisme. J'ai raté tous mes concours pour monter dans la hiérarchie de la compagnie de l'Opéra. J'ai développé une habilité dans l'escamotage du ratage, faire croire que tu fais quatre pirouettes alors que tu n'en fais que deux, tourner une fois en l'air au lieu de deux sans que cela se voie en agitant la tête. Apprendre à donner l'illusion d'un geste que tu ne fais pas, c'est aussi cela que la danse classique m'a appris. Quand j'ai compris cela, j'ai commencé à être un danseur contemporain, et à me défaire d'une pensée classique.

A. P. : Peut-on construire un spectacle à partir d'un processus de ratage systématique ? Du spectacle intitulé Morceau fait avec Latifa Laâbissi, Jennifer Lacey, Yves-Noël Genod, tu dis qu'il s'est construit "sur les ruines de nos expérimentations », que vous avez cherché à faire " une suite pour matériaux faibles ${ }^{2}$ ». Le projet était, et je te cite dans un entretien de l'année dernière, "d'avoir de faibles idées, dans un corps affaibli, avec un faible désir d'atteindre ${ }^{3}$ ». Dans Morceau, l'idée est de s'intéresser «à tout ce qui ne marche pas », et travailler dans les zones d'affaiblissement et non de puissance.

L. T. : Je repars un tout petit peu en arrière pour dire comment Morceau arrive dans ce parcours. Entre 1997 et 2000, j'interroge les rapports qui s'engagent entre scène et salle. Je m'intéresse aux conditions de la rencontre entre l'œuvre et le public. Comment la communication que fait le théâtre, le programme de la pièce, les conventions du rituel de représentation - lever de rideau, entrée en scène, lumières, scénographie, durée, frontalité, architecture du lieu, tous ces paramètres qui organisent la réception de l'œuvre - sont autant de filtres pouvant faire écran à celle-ci. Je cherche un geste partagé entre celui qui le fait et celui qui le voit et j'ai l'impression que les attentes du public sont augmentées par ces paramètres. Je ne sais ni y répondre ni passer outre. La seule solution que je trouve est de fuir le théâtre.

Dans ma fuite, je rencontre l'artiste visuel Francisco Ruiz De Infante. Une de ses préoccupations concerne la place et le contrôle du public dans la circulation de ses installations. Équipé de caméras et de cellules photo-électriques, il organise à leur insu la trajectoire des spectateurs dans les salles d'exposition, conditionnant leurs déplacements et excitant leurs états de réception. J'avais l'impression d'être contrôlé par le spectateur alors que Francisco Ruiz De Infante, lui, inverse le rapport et contrôle le spectateur. Ensemble, on va imaginer plusieurs projets, et notamment Souvent dans la forêt... (1997) à Consonni, friche industrielle insalubre de $8000 \mathrm{~m}^{2}$ à Bilbao, au bord de la Ria, dans un quartier en devenir dans lequel se construit le Guggenheim. On choisit de faire une déambulation dans l'espace de cette friche pour 120 personnes qu'on invite à venir à $5 \mathrm{~h}$ du matin, en leur faisant traverser la Ria en bateau. On les fait rentrer dans la friche par petits groupes en les menant, au point de 
les perdre, d'improvisations en improvisations, un peu inquiétantes, un peu délirantes. Ce parcours de deux heures laisse le public hagard, dans un état de suspension. La sensation d'avoir assisté à quelque chose qui n'existe pas était palpable, un état d'inquiétude circulait entre performeur et public. Cela va être une expérience fondatrice pour nous. Enfin se partageait véritablement le lieu, le geste, la durée, l'imaginaire par le risque pris de part et d'autre. Nous allons transposer ce projet dans le centre d'art de la Ferme du Buisson (Noisiel) avec une reprise pour 19 spectateurs, au galop et dans une sorte d'hystérie, dans lequel on va imposer au public une situation de grand délire paranoïaque. Nous proposions un dispositif où le public se retrouvait allongé dans des lits de camps, assis sur des chaises accrochées sur des murs à un mètre du sol, enfermé dans des espaces exigus dans le noir et finalement jeté sur un parking à la fin du parcours. On va recevoir des lettres d'insultes, d'étonnement ou d'effroi même! Je commençais à comprendre comment on pouvait bouger le corps du public, comment cela générait une dynamique. Nous n'étions pas dans ce que l'on appelle un "projet participatif». Les matériaux et situations que nous proposions étaient autant produits par les performeurs que par l'inquiétude du public face à la chose regardée, mettant en tension l'exposition des uns et des autres. Ça a complètement éventré les notions traditionnelles que j'avais du spectacle. Je ne pouvais plus aller faire une pièce de danse sur un plateau. J'ai acheté un chapiteau. L'espace performatif est devenu une toile migrante, déplaçable, pliable, dans lequel on reconstruisait une scène. Nous sommes partis en tournée dans des territoires ruraux, les gens qui venaient nous voir étaient assommés, ils ne comprenaient rien à ce qu'on faisait. Et nous on était dans des états seconds, on faisait des propositions dadaïstes, déstructurées, poétiques et incompréhensibles.

Toutes ces expérimentations ont duré trois ans. Nous proposions au public des situations improbables et des places inconfortables pour voir la danse. Mais quelque chose résistait, je considérais que nous n'arrivions toujours pas à partager l'imaginaire du geste.

Notre langage avait bougé, nos danses s'étaient nourries de ces déplacements mais nous restions virtuoses et cette virtuosité laissait le public à une certaine distance. Il continuait d'assister plus que de venir combler par leurs imaginaires ce que nos danses ouvraient comme espace pour eux.

J'ai alors compris qu'il ne fallait pas déserter les théâtres. La scène était probablement le meilleur lieu pour faire spectacle. À un moment, j'ai compris que ce n'était plus déplacer le public qui était important, mais la nature du matériau exposé qu'il fallait interroger, sa validité. Comment son affaiblissement pouvait permettre une reconfiguration de la distance entre celui qui fait un geste et celui qui le reçoit, comment cette distance se mesure en désir de voir. Plus le geste a une sorte de faiblesse, d'inachèvement, voire d'échec, dans le sens où le regard du spectateur peut venir échouer dans le geste, plus l'imaginaire est partagé entre celui qui fait le geste et celui qui le reçoit.

Morceau a d'abord été un long processus avant d'être présenté comme une pièce. Nous n'avons pas cherché à travailler sur la notion de ratage, on est partis d'une observation. Jusqu'alors, quand on présentait une pièce, elle était toujours le fruit de ce que nous avions fait de mieux dans nos expérimentations. Nous exposions toujours la meilleure chose que nous avions produite et travaillée. Cela supposait aussi que 
certains matériaux n'avaient pas été conservés car considérés comme moins valides, moins réussis. Alors nous avons inversé le processus. Tout ce qui nous semblait valide nous l'écartions et tous ce que jusqu'alors nous jetions à la poubelle devenait le matériau à exposer. Si on veut élargir nos territoires esthétiques, de pensée et de forme, il faut qu'on se dégoûte, dans le sens de déborder de son goût, faire confiance à des matériaux plus fragiles, moins efficaces, avoir la liberté de pouvoir utiliser tout type de matériaux, parler, chanter, raconter des histoires, manipuler des objets, tout ce qui faisait récit même si ce récit semblait pauvre et peu productif. On s'est beaucoup amusés avec une immense permissivité. Le processus de travail partagé avec Yves-Noël, Latifa et Jennifer, a consisté à passer chacun notre tour devant les trois autres. Nous faisions de longues improvisations à partir de très peu de choses, de peu d'idées, pendant des jours sans jamais avoir de retour. On ne savait pas ce que les autres avaient pensé. Éventuellement on pouvait faire des feed-back performés en reprenant le matériau vu chez l'un.e ou l'autre. Puis on dînait tous ensemble et chacun discrètement essayait de savoir ce que les autres avaient pensé. Une sorte de conversation s'instaurait, rien de direct, comme des confidences hors du temps de travail. Morceau est né de ce processus très libre, avec quelques contraintes simples. Par exemple: nous devions entrer sur scène et sur une durée qui ne devait pas excéder une minute, faire une seule chose, une situation, une danse, une proposition faible en nous tenant à 3 mètres du public au centre de l'espace, de face. A cette place la plus évidente, celle que nous nommions de «l'impact populaire».

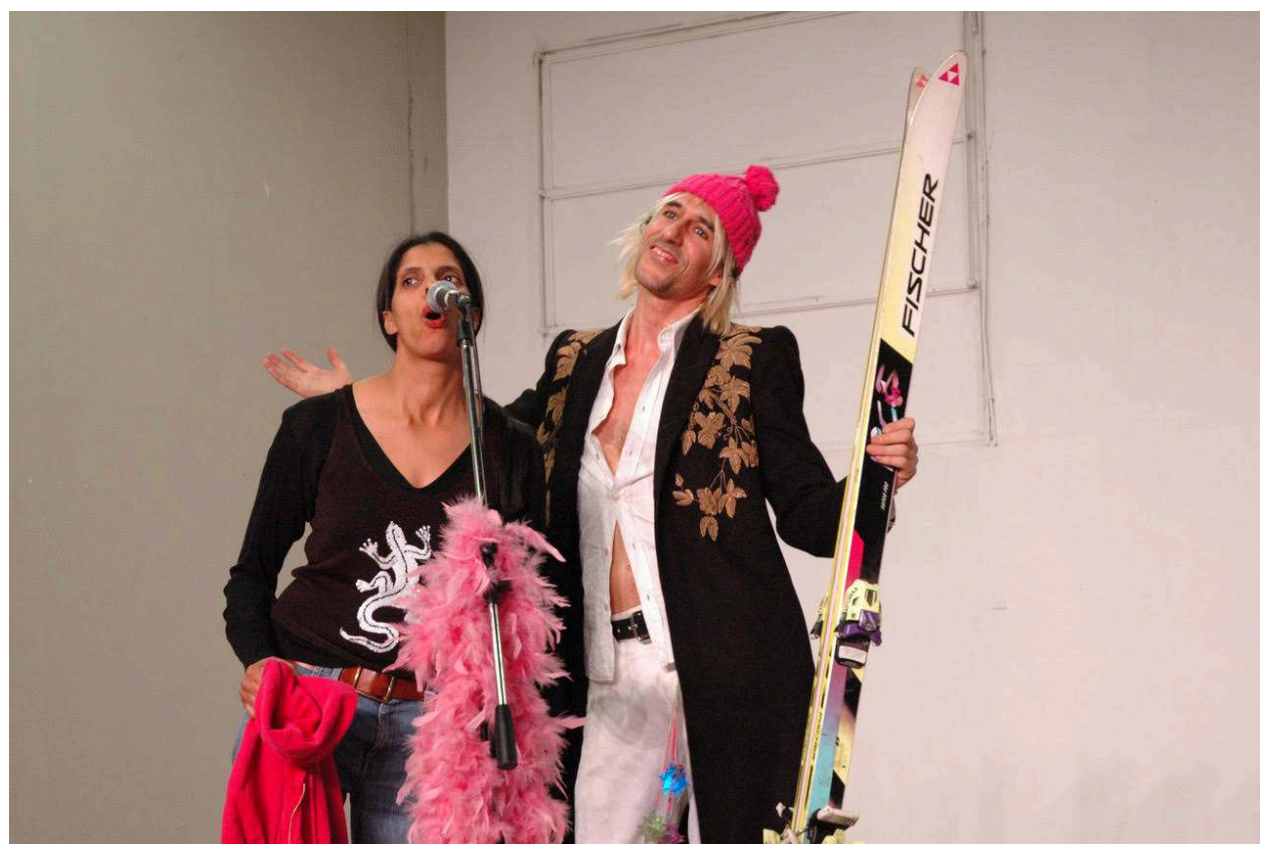

Latifa Laâbissi et Yves-Noël Genod dans Morceau

(c) Cosimo Terlizzi

A. P. : II n'y a donc pas de superposition entre faiblesse et raté. On change d'échelle de valeur. Ce qui est normalement rebus va devenir la chose à mettre en avant. La question du ratage, au sens de manquer son but, n'intervient plus dans ces cas-là.

L. T. : Morceau participe tout de même d'une esthétique du ratage. Chaque personne vient devant le public pour tenter quelque chose qu'il n'arrive pas à faire. On parlait de sabotage. Collectivement on essayait de faire en sorte que le spectacle s'effondre, et individuellement on devait sauver le spectacle. Ce paradoxe créait une sorte 
d'excitation que le public partageait avec visiblement beaucoup de plaisir. La réjouissance, elle était là, dans l'amplification du désastre par la tentative du sauvetage même. L'esthétique du travail était le déficit, les propositions étaient déficitaires. Non seulement on manquait la cible, mais on pouvait même se demander s'il y avait une cible qui avait été pensée. Donc, pas de raté s'il n'y a pas de cible. Mais quand même, on faisait croire qu'il y avait une cible, et qu'on la ratait.

A. P. : Ça travaille une autre dimension du ratage. Quand on dit de quelqu'un que c'est un raté, on parle de quelqu'un qui n'a pas d'objectif dans la vie, pas de cible, pas de but? Présenter un spectacle qui n'a pas de cible, c'est poser une esthétique du ratage, dans le sens d'errance, de non but.

1 L. T. : Yves-Noël danse avec une non-connaissance de l'académisme chorégraphique contemporain et classique. Pour moi qui venais avec mon corps ultra conditionné, formaté, produit d'une expérience patrimoniale, je vois dans ses gestes une puissance de danse très rare. Ce que j'appellerais la grâce réelle du danseur, sans le formatage technique. Ce que j'admire à ce moment-là, et que je vois quand il danse, c'est quelqu'un qui tente de faire du mieux possible une chose qu'il ne sait pas vraiment faire. L'espace entre son désir de faire le geste qu'il ne sait pas et la réussite de celui-ci, c'est son imaginaire qui le comble. C'est la grâce absolue de la maladresse qui devient style. Je vais apprendre beaucoup à ses côtés. Je tente à cette époque de me défaire d'une technique encombrante, je cherche à m'approcher de ce que je vois dans ses danses. Avec Morceau, on va s'intéresser au peu d'effet, au pas grand-chose, au presque rien. Un petit récit sans grand intérêt, une petite histoire sans chute, une pauvre petite danse dans un coin, un objet inanimé qui soudain commence à bouger par la grâce du très peu. On va faire monter ces matériaux vers leur dimension poétique. Cette poésie va rester une poésie du pas grand-chose. Mais le public va se mettre à jouer avec nous. Un climat de connivence va s'instaurer. Jusqu'à présent, je ne supportais pas cette connivence. Mais avec Morceau on va se mettre à parler directement au public, et on va lui faire croire qu'on est complice. Mais on va garder la main, on va flirter avec le mauvais goût, la chose qui ne se fait pas. Plus la pièce était éventrée, plus le public mettait de l'énergie pour rassembler les morceaux et plus la pièce réussissait de son échec. Il y avait une grande joie à ça. Ça a duré six ans, dans chaque théâtre où nous étions programmés nous arrivions avec un sac de matériaux déjà utilisés précédemment et avec un processus exploratoire qui permettait d'en réinventer in situ. À chaque lieu, des matériaux nouveaux surgissaient. Le processus était collectif, l'apport de chacun déterminant. Ça a été une matrice très importante où j'ai eu l'impression de renverser la table dans ma manière de composer et de considérer la scène. 


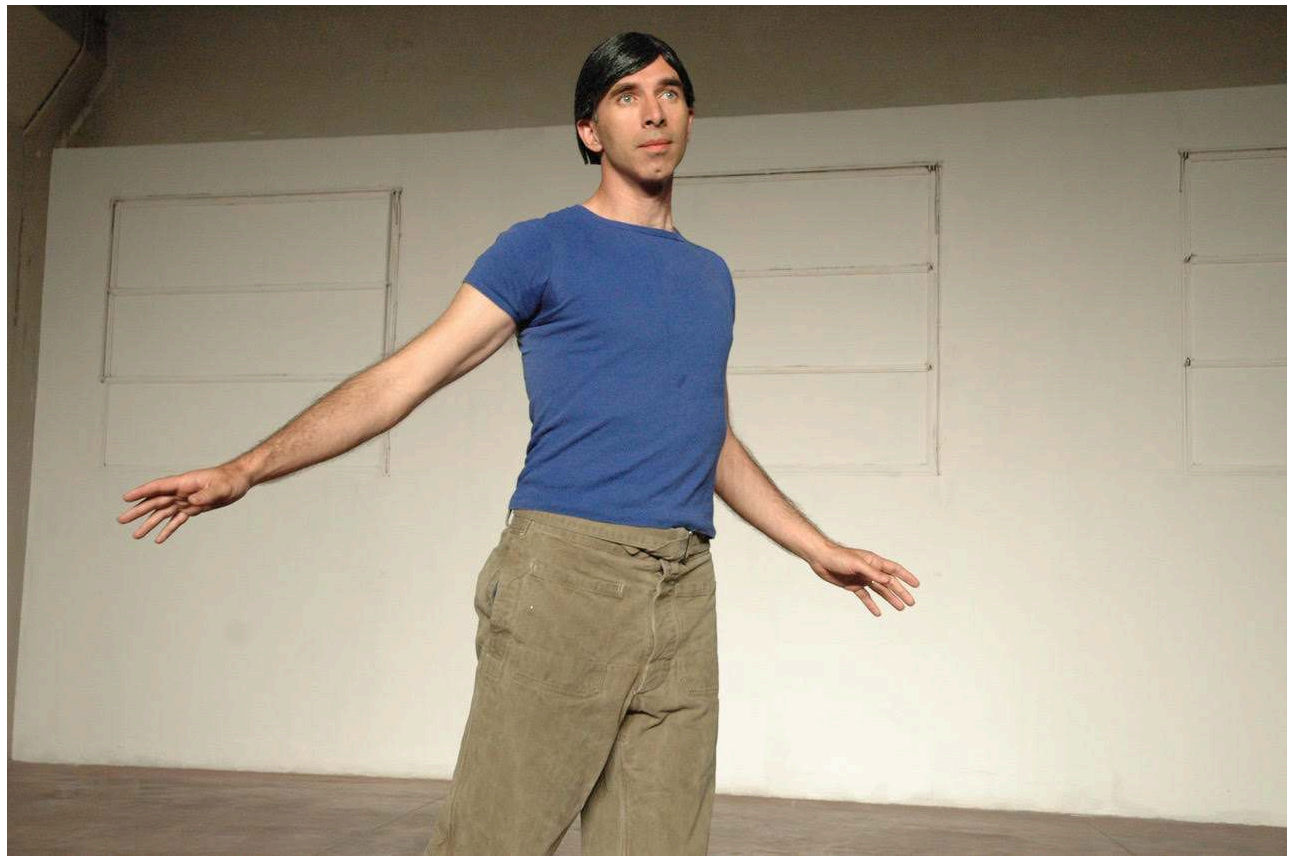

Loïc Touzé dans Morceau

(c) Cosimo Terlizzi

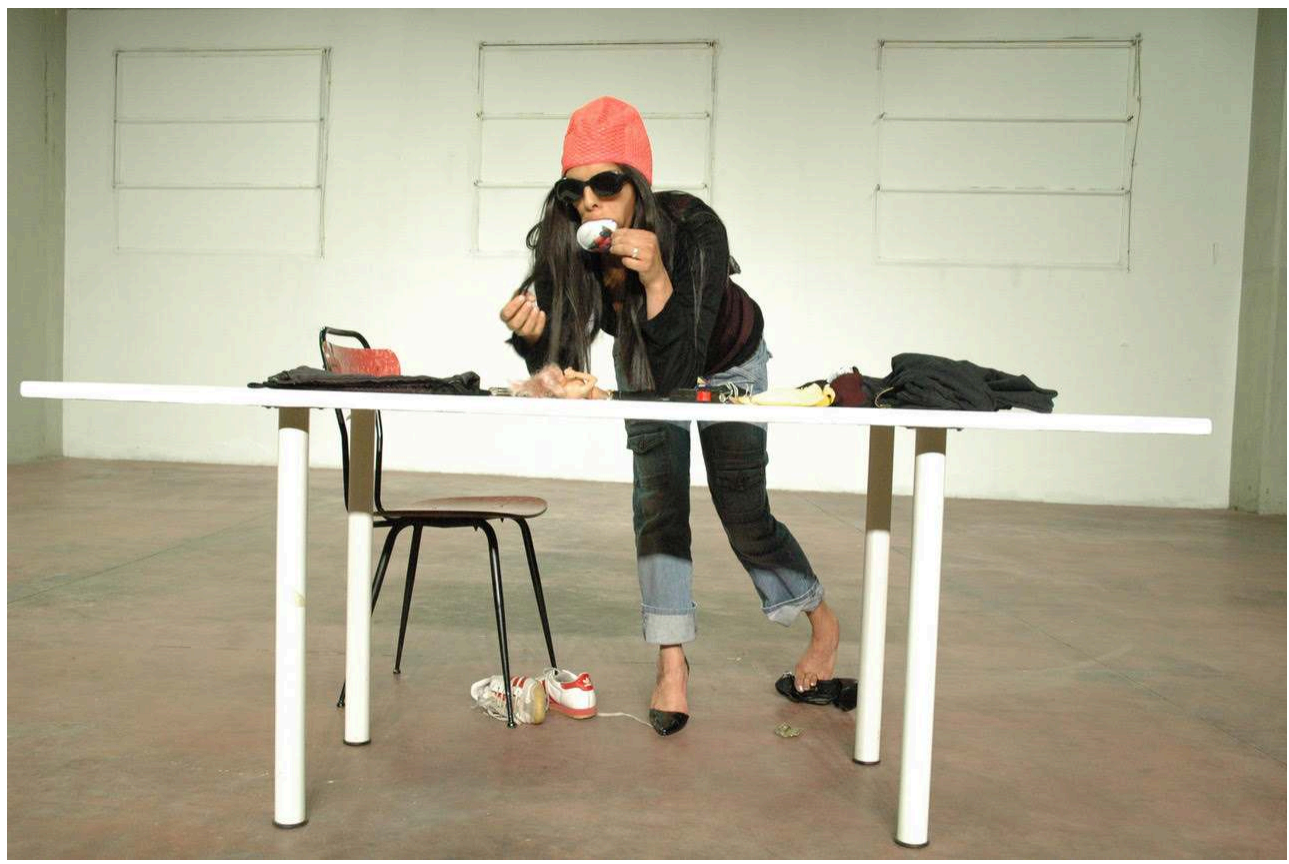

Latifa Laâbissi dans Morceau

(c) Cosimo Terlizzi

A. P. : Est-il important de revendiquer le ratage, soit comme mode de fabrication, soit comme droit dans un parcours de production? Est-ce que tu revendiques, dans notre société néo-libérale, le droit au ratage, si on l'entend comme la possibilité de l'essai et de l'échec, et est-ce que cela a des implications politiques?

L. T. : J'ai commencé véritablement à faire mon travail de chorégraphe à partir du moment où j'ai compris que je n'étais pas obligé de réussir. Tant que je suis engoncé, psychologiquement et économiquement dans un système où il faut que je réussisse, 
c'est-à-dire que je sois productif, cela ne fonctionne pas. Il m'a fallu un certain temps pour résister aux attentes du système de production, des lieux, tutelles, expertises.

Je vais trouver des stratégies non pas pour réussir mais pour avoir le temps de faire la pièce que $\mathrm{j}$ 'ai envie de faire. Le temps et l'économie de production autorisent rarement un processus de ratage, d'échec, alors qu'il est indispensable de se tromper, de se perdre, de passer par des chemins inédits, de remettre en question tout le processus, de changer de projet même. Je réalise assez peu de projets déjà écrits.

La réalisation du projet, dans le sens de "devenir réel ", se fait au moment où il est exposé, dans la manière dont il est perçu, reçu par le public.

J'ai compris que je pouvais changer de paradigme en donnant la même valeur à la notion de représentation et à la notion d'expérience. Dans le processus que j'engage, les interprètes avec qui je travaille augmentent leur expérience, et cette augmentation va jusqu'à produire une augmentation de l'expérience pour le spectateur.

A. P. : Quelle est la place que tu donnes au raté et au ratage dans ton travail de pédagogue ? Tu dis qu'il n'y a pas d'apprentissage sans ratage. Est-ce que c'est audible dans tes interventions dans les écoles?

L. T. : L'idée de réussite est absente de mon enseignement. La notion principale sur laquelle est fondée ma pédagogie est l'expérimentation dans le sens de la tentative, de l'hypothèse, du risque, de la découverte. Je ne valorise aucunement la notion de réussite. Elle arrive, mais elle ne résout rien des questions que nous partageons. Elle n'est pas un but, elle peut momentanément être un repaire mais reste complètement instable. Rater est plus tangible et offre une plus grande dynamique pour les questions posées. Nous conspirons ensemble. Je substitue par l'imaginaire les idées d'excellence et d'attente. Nous créons avec mon ami et collaborateur Mathieu Bouvier des dispositifs dans lesquels la question n'est plus de réussir quelque chose, mais de jouer à quelque chose. Quand il y a une injonction de résultat la tension s'installe et l'imaginaire se retire. L'imaginaire ne peut prendre place que dans des espaces tendres, calmes et sans attentes. Je vais prendre soin de défaire les relations implicites de domination que l'espace de formation charrie. C'est par des fictions partagées que s'inventent des rapports nouveaux, des lieux, des actions. Je ne porte pas de projet éducatif dans mon enseignement, je viens avec l'idée que si nous sommes ensemble c'est pour découvrir et développer nos dons. L'observation est un des outils les plus opérants. Je suis très inspiré par la méthode Feldenkrais. Cette pratique somatique ouvre vers une prise de conscience corporelle plus large que le schéma dans lequel nous sommes souvent enfermés. Le processus pédagogique est celui-ci : élargir, déplier, envisager, voir en deçà et au-delà de ce que le geste dansé contient. Faire confiance en l'effectivité de ce geste, en sa puissance, en sa capacité à ouvrir des perceptions plus larges pour saisir le réel, agir avec lui.

A. P. : Tu parlais de la manière dont tu arrivais dans les institutions, et je sais que tu vas intervenir avec les Ballets de Lorraine. Comment tu vas travailler dans cette institution-là, pour les amener à un autre geste, peut-être un geste maladroit ?

L. T. : L'expérience que j'ai faite avec la danse classique et la déconstruction que j'ai opérée pour sortir de ce schéma corporel imposant est une aide pour venir travailler avec les danseuses et danseurs du Ballet de Lorraine. Je ne vais pas leurs demander de se défaire de leur compétence, je vais plutôt les inviter à en reconnaitre toute la richesse. Parallèlement, je vais leur suggérer de faire un pas de côté en attirant leur 
attention sur le fait que cette puissance, ces savoirs acquis, ne servent à rien s'ils ne sont pas là pour faire l'expérience d'un geste inédit. Je vais les guider vers une augmentation de leur langage de danseurs, ouverts vers d'autres sensations, d'autres imaginaires de la danse. La pièce que nous allons composer ensemble n'exposera pas leurs compétences. Le théâtre qui m'occupe n'est pas fait pour cela. La danse demande de l'espace, un risque pour apparaitre. Si les compétences prennent toute la place, la danse s'absente. L'écriture du projet va nous amener à découvrir cela.

Je cherche aussi à partager avec eux l'idée d'une danse sans effort. C'est compliqué cette affaire, car l'effort est un retour d'expérience direct pour le danseur. S'il y a de l'effort, il se sent exister dans son geste. Je les invite à explorer un geste plus dilaté, plus relâché, plus abandonné, mais en même temps sans répit dans la concentration, l'observation et l'attention. Ce sont des formes de débordement de soi qui ne sont ni des formes d'excellence, ni des formes de dépense. La maladresse ne rôde pas très loin. La difficulté la plus grande avec ces interprètes, c'est qu'ils sont très habiles et très rapides pour répondre à toutes les sollicitations. Ils sont engagés pour cela, pour s'adapter rapidement à toutes formes de langage chorégraphique.

Mais la manière dont ils abordent ces différents langages est souvent identique. Je dois être inventif pour créer les conditions d'une impossibilité de répondre correctement, pour que les questions que nous allons rencontrer restent dynamiques, ouvertes, fertiles pour eux et pour nous qui allons les voir danser.

A. P. : Mais tu ne peux pas non plus venir à eux en leur enjoignant de rater. La grâce du ratage ne se décrète pas.

L. T. : Elle ne se décrète pas, mais elle s'observe. Mon travail, il est précisément là : faire voir au danseur comment sortir de son phrasé, de ses patterns, de son éducation, de sa culture du mouvement. Cela passe par assumer de l'hésitation, des changements d'investissements toniques, des appuis différents, des pré-gestes inédits, des gestes sans résolution, prendre le risque de la maladresse. Alors, par ces investigations, quelque chose jusqu'alors négligé se met à croître, se transforme. Ce sont les mots échangés qui sont les bords et les contours de l'expérience. Ces mots, nous devons les trouver, les partager. Ce sont les clés pour accéder à un hors de soi, à une sortie du langage connu. Il y a souvent de la confusion quand on traverse ce processus, un brouillard. Inventer une danse passe aussi par apprendre à survivre à cette confusion.

Le titre du spectacle est No oco, ce qui veut dire « au creux » en portugais. En fait, je désigne un lieu pour leur propre geste. Leur danse à eux, est le plus souvent pleine de muscles, d'atteintes, de directions, d'ordres. Proposer d'aller au creux, cela désigne aller au-dessous, à l'intérieur, en deçà, quelque part entre leur geste et eux-mêmes, à l'endroit de l'intention. Il y a souvent un écart, une perte entre l'intention et l'action. Il y a là un lieu étonnant qu'il est nécessaire d'identifier et de nourrir. Rater ou réussir se tient là comme en embuscade. C'est l'endroit où «je » existe. Ce «Je » est plus large, plus inconnu, il n'est en rien personnel. Comment me tenir là, au début des choses, au commencement, avant toute production de signe. Je me porte et me tiens là pour que le geste soit une aventure inouie, non pas pour réussir ce que j'ai à faire mais échouer à nouveau autrement, « échouer mieux ». 


\section{NOTES}

1. Groupe de Recherche Chorégraphique de l'Opéra de Paris.

2. «Morceau s'est construit sur les ruines de nos expérimentations. Conçu comme une suite pour matériaux faibles. Dans ce que nous trouvions lors de nos recherches, nous avons chaque fois privilégié ce qui nous semblait le moins valide, le moins beau, le moins présentable et on s'est mis à composer avec ces rebuts. Toutes les tentatives que l'on refoule habituellement devenaient notre trésor de guerre. On a pris grand soin de ces "bas morceaux », on a pratiqué et cultivé ce goût du mineur ». In Bouvier Mathieu, Touzé Loïc, Le grain de l'image. Entretien avec Loïc Touzé, in www.pourunatlasdesfigures.net, dir. Mathieu Bouvier, La Manufacture, Lausanne (He.so) 2018, consulté en ligne le 11/06/2020.

3. https://www.duuuradio.fr/episode/la-danse-de-quelquun-dautre-que-soi

Voir par exemple le $2^{\mathrm{e}}$ podcast: «La prise de conscience d'un geste autre de celui qui m'a été inculqué, c'est de voir la grâce d'une maladresse. C'est de voir tout d'un coup hors des canons qui étaient l'exactitude, la virtuosité, la spécificité d'un geste réussi qui était ce modèle-là vers lequel on devait tendre, j'ai été bouleversé de voir quelqu'un vouloir faire du mieux possible un geste qu'il n'arrive pas à faire (...) je trouvais incroyable que l'on pouvait négliger la maladresse alors que dans cette maladresse le désir de faire était plus fort ».

\section{INDEX}

Mots-clés : carrière, pédagogie, déconstruction, faiblesse

\section{AUTEURS}

\section{LOÏC TOUZÉ}

Loïc Touzé est danseur, chorégraphe et pédagogue. S'il crée, depuis les années 90 , des pièces toujours en collaboration avec les interprètes qui l'accompagnent (Morceau, Love, La Chance, Fanfare, Forme Simple), ses créations peuvent s'incarner dans des formats très divers : un protocole urbain autour du geste (Autour de la table, coécrit avec Anne Kerzerho), un film (Dedans ce monde), une conférence performée (Je suis lent). Chercheur associé à la Manufacture, il a participé ou participe à un certain nombre de programmes de recherches. Il est intervenant dans de nombreuses écoles d'art (Exerce, Écoles du Théâtre National de Strasbourg et de la Manufacture de Lausanne). Il dirige depuis 2011 Honolulu, lieu de travail à Nantes pour la création contemporaine.

\section{ANNE PELLOIS}

Anne Pellois est maîtresse de conférences en Études théâtrales à l'École normale supérieure de Lyon. 